\title{
Spina Ventosa in a Young Adult: Report of Two Gases
}

\section{JCR Vishnu Senthil, Anil K Bhat, Ashwath Acharya}

Department of Orthopaedics, Kasturba Medical College, Manipal-576104,

Karnataka, India.

\section{Abstract:}

Tuberculous dactylitis is a rare extra-pulmonary manifestation common in children. Radiological and clinical presentation of tuberculous dactylitis creates confusion in diagnosis and delays treatment, which could lead to progressive joint deformity and loss of function. Two patients presented with pain and swelling of the hand accompanied with a discharging sinus in one of the patient. Radiologically both patients presented as a lytic lesion without any evidence of a primary foci. Culture and microscopic examination confirmed tubercle granuloma in the lesion. Both patients were started on anti-tuberculosis drugs and one patient underwent ray amputation. At final follow up, both patients had good hand function.

Key words: Amputation, Bone Diseases, Granuloma, Pain, Tuberculosis.

\section{Introduction}

Tuberculosis (TB) is the second most common cause of skeletal infection in the developing world. Bone and joint tuberculosis constitute 1\%-3\% of extra-pulmonary TB. Spine is commonly affected. Tuberculous dactylitis (spina ventosa) is a rare extra-pulmonary manifestation with incidence of $0.6 \%-6 \%$ in children. It commonly occurs in children less than six years which constitute $85 \%$ of cases. We report two cases of tuberculous dactylitis in adults and the diagnostic dilemma behind it.

\section{Case Report}

\section{Case 1:}

35 year old lady presented with right middle finger swelling and pain for six months duration [Fig.1].
Swelling was insidious and progressive. No history of trauma, fever or constitutional symptoms could be elicited. On examination, the middle finger was swollen with firm to hard in consistency. Movements of the proximal inter-phalangeal and middle inter-phalangeal joint were restricted and painful. Sensations were reduced distally, capillary refill was less than $3 \mathrm{sec}$ and no evidence of discharging sinus was present. Clinical diagnosis of chronic tenosynovitis, osteomyelitis and benign tumor were considered. Radiological evaluation showed a lytic lesion with destructed of both dorsal and volar cortex of the proximal phalanx. There was a loss of height of proximal phalanx. Honeycomb appearance without any evidence of sclerosis further added to the diagnostic dilemma [Fig.2]. Chest X-ray

\section{Corresponding Author: Dr. Vishnu Senthil}

Email: vishsnake@gmail.com

Received: May 30, 2016 | Accepted: July 15, 2016 | Published Online: September 20, 2016

This is an Open Access article distributed under the terms of the Creative Commons Attribution License (creativecommons.org/licenses/by/3.0)

Conflict of interest: None declared | Source of funding: Nil | DOl: http://dx.doi.org/10.17659/01.2016.0108 
showed no evidence of active or healed lesion [Fig.3A]. Radiological diagnosis of enchondroma was considered but other pathologies like giant cell tumor, fibrous dysplasia, aneurysmal bone cyst and chronic osteomyelitis were also considered as differentials. Blood investigations showed an ESR of $27 \mathrm{~mm} / \mathrm{hr}$, CRP of 1.6 and total leucocyte count of $6,400 / \mu \mathrm{L}$ with other investigations in a normal range. Ultrasound showed well defined fluid collection around the proximal phalanx suggesting post-infective pathology. Open excisional biopsy of entire phalanx was done. Intra-operative findings suggested granulomatous tissue around the phalanx with fibrosis of flexor sheath and tendons. Complete fibrotic involvement of the soft tissue and digital neurovascular structures was present intra-operatively. Histo-pathological examination

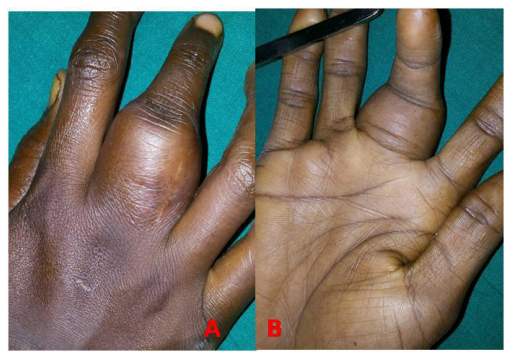

Fig. 1: Fusiform swelling of the proximal phalanx of middle finger $[A]$, dorsal $[B]$, volar surface.

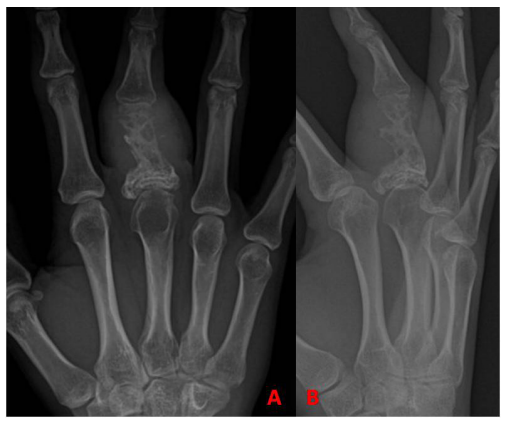

Fig.2: Radiograph of hand anterior-posterior [A] and oblique [B] showing lytic lesion in the proximal phalanx of middle finger with honeycomb appearance. showed caseating granulomas composed of giant cells, macrophages and lymphocytes with congested blood vessels suggesting tuberculous granuloma [Fig.4]. In view of extensive destruction of the flexor and extensor tendons, patient was given the option of staged reconstruction or ray amputation. She chose the latter due to financial reasons [Fig.5]. Patient was put on anti-tubercular therapy (ATT) post-operatively with an intensive phase of four drugs of isoniazid, rifampicin, pyrazinamide, ethambutol and pyridoxine supplementation every day for two month duration. Continuation phase of isoniazid and rifampicin was given for nine months. Serial ESR was performed monthly and regimen was stopped when three consecutive ESR were $1 \mathrm{~mm} / \mathrm{hr}$. At twenty four months follow up, patient is having good hand function with no evidence of recurrence.

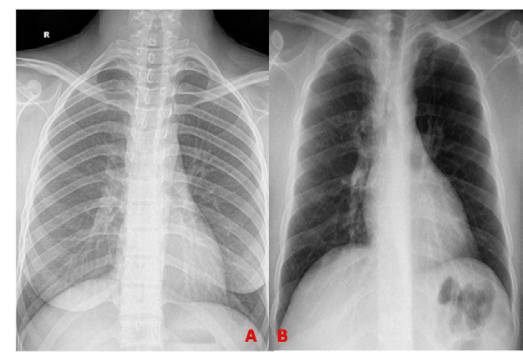

Fig.3: Radiograph of chest showing bilateral clear lung fields in case 1 [A] and case 2 [B].

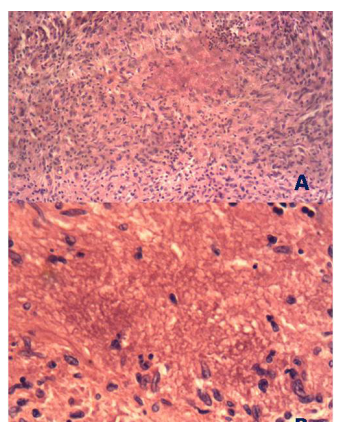

Fig.4: Microscopy of low power 10X [A] and high power 40X [B] sections showing central necrosis with lymphocyte infiltrate. Epitheloid granuloma consisting of Langhans and foreign body giant cells are seen. 


\section{Case 2:}

33 year old male presented with discharging sinus over the dorsum of left hand near $3^{\text {rd }}$ web space for two months duration [Fig.6A]. No history of open injury, fever or constitutional symptoms or any co-morbidities were elicited. On examination, a sinus of $2 \times 1 \mathrm{~cm}$ fixed to underlying bone was present over the dorsum of third web space. Discharge was serous and foul smelling. On examination, no localized warmth or tenderness was present. Movements of third metacarpo-phalangeal joint were restricted. Sensation and capillary refill was normal. Other joints were clinically normal. Radiological investigation showed a lytic lesion involving the third metacarpal epiphysis and extending into the metaphyseal region [Fig.6B]. Clinical diagnosis of bone cyst, giant cell tumor and infection (osteomyelitis) were considered. Blood investigations showed an elevated ESR of $30 \mathrm{~mm} / \mathrm{hr}$, CRP of 0.6, total leucocyte of 10,000 $\mu \mathrm{L} / \mathrm{ml}$. Culture swab from the sinus revealed only pus cells with no evidence of bacterial growth. Patient was empirically started on third generation cephalosporin. Chest $X$-ray revealed no evidence of a primary focus [Fig.3B]. Pain was worsening inspite of antibiotic coverage. Patient underwent incisional biopsy and the curetted material resembled unhealthy pale granulation tissue. Microscopic examination showed foci of necrosis surrounded by palisading layer of epitheliod cells, foreign body and Langhans giant cells. Lympho-plasmacytic and eosinophilic infiltrates were present suggesting granulomatous inflammation [Fig.4]. PCR was positive for Mycobacterium tuberculosis. Patient was started on anti-tuberculosis drugs with an intensive phase of 2 months of four drugs consisting of isoniazid, rifampicin, pyrazinamide and ethambutol every day and nine months of continuation phase with isoniazid and rifampicin. Serial ESR was performed monthly and the continuation phase was stopped when three consecutive ESR were $1 \mathrm{~mm} / \mathrm{hr}$. Patient now at two years follow up, with no episodes

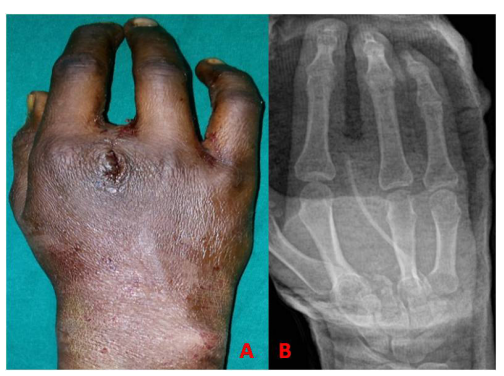

Fig.5: Clinical picture [A] and radiograph [B] showing ray amputation of middle finger with good hand function.

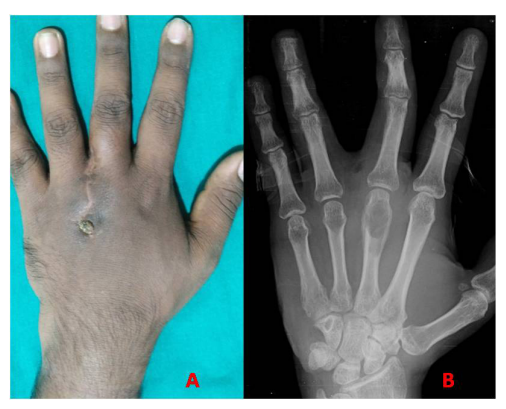

Fig.6: Clinical photograph [A] and radiograph of hand $[B]$ showing discharging sinus on the dorsum of hand with well-defined expansile lytic lesion in meta-diaphyseal region of distal shaft of $3^{\text {rd }}$ metacarpal with narrow zone of transition and associated with a thin single layer of lamellated perosteal reaction with cortical breach.

of discharging sinus and presence of good hand function.

\section{Discussion}

Bone and joint tuberculosis occurs in spine $(51 \%)$, pelvis $(12 \%)$, hip and femur $(10 \%)$, knee and tibia $(10 \%)$, ribs $(7 \%)$ and involvement of small bones of hand and feet is rare [1]. In 1803, Boyer gave the anatomical description: spina ventosa (spina: short bone; ventosa: inflated with air). In 1837, Nelaton proved the etiology. In 1886, Rankin identified TB dactylitis by histological technique. Fieilchenfeld in 
1896 described TB dactylitis roentgen-graphically in children $[2,3]$.

Hand is frequently involved than foot. Proximal phalanx of the index and middle finger is commonly involved. Spina ventosa is a spindle shaped expansion of tubular short bone causing absorption of cortex, bordering the medullary canal with subperiosteal hyperplasia and new bone formation. Abscess and sinus formation is not uncommon. Sclerosis may occur in long standing cases [4]. Children are affected due to rich metaphyseal blood supply of the phalanx. Occlusion of central nutrient artery by the bacteria produces sequestra and forms the tuberculosis granuloma [5]. TB disease runs a protracted course from a few months to $2-3$ years which leads to deformity and stiffness of joint.

In our reported cases, the causation of TB dactylitis could be direct inoculation or hematogenous spread of bacilli due to Indian sub-continent being endemic for tuberculosis. Tubercle bacteria are present in soil which could be inoculated by trivial trauma like abrasion. Hand being in contact with the external environment continuously is subjected to trauma easily. Presentation of tuberculous dactylitis in adults opens up a diagnostic dilemma, often mistaken for chronic osteomyelitis, enchondroma, fibrous defect and other granulomatous condition. Literature review of TB dactylitis, reported that 41 out of 60 cases occurred in individuals less than 20 years of age $[6,7]$.

Radiological findings are often confusing because of varied presentations like cystic or lytic lesion. Lesion may also present with cortical erosion, cortical destruction, periosteal reaction, sclerosis, sequestrum or with pathological fracture. In pyogenic infection, destruction is more profund and presents with systemic symptoms. Sarcoidosis presents as a well demarcated cystic lesion with no bony expansion or periosteal reaction [8-10]. In both the cases TB presented as lytic lesions and clinic-radiologically was considered as pyogenic osteomyelitis but TB could be discovered only in histopathological diagnosis.

Diagnosis of tuberculosis should fulfill either of the following, positive culture or histological findings of caseous granulomas with clinical response to anti-tuberculosis drugs [11]. Response to anti-tuberculosis drugs which is given in divided phases is good. Healing of sinus and lesions are seen both clinically and radiologically. Dedicated rehabilitation protocol is implemented during the treatment phase to prevent joint stiffness. Surgical management is considered when there is permanent deformity or arthritis of joint.

Our both cases were incidentally diagnosed as tuberculosis only at the stage of biopsy. Diagnosis was missed in the initial stages due to lack of suspicion of this common and widespread infection in our endemic country. Rise in immunosuppressive conditions like HIV has increased the presence of $T B$ in uncommon sites and age. The only high risk factor was belonging to a lower socio-economic status which could have led to easy inoculation and spread of TB bacilli. Pyogenic osteomyelitis or a benign tumor was kept as a main differential diagnosis. Elevated ESR in both our cases should have led to suspicion to TB. After a proven biopsy the patient was started on ATT and the response was monitored with serial ESR and X-rays.

\section{Conclusion}

Spina ventosa is a rare manifestation of skeletal tuberculosis in adults. Awareness of this uncommon presentation of a common disease in adults is needed to prevent delay in diagnosis and treatment. Tuberculosis should always be considered as a differential diagnosis in lesions that present as diagnostic dilemma because of non-specific clinical features and occurrence in uncommon sites in an endemic region. 


\section{References}

1. Panchonia A, Kulkarni CV, Mehar R, Mandwariya S. Isolated Tuberculosis Dactylitis (Spina Ventosa) in a 9 year old boy - A Rare Entity. International Journal of Basic and Applied Medical Sciences. 2012;2(2):52-55.

2. Pearlman HS, Warren RF. Tuberculosis dactylitis. AM J Surg.1961;101:769-771.

3. Subasi M, Bukte Y, Kapukaya A, Gurkan F. Tuberculosis of the metacarpals and phalanges of the hand. Ann Plast Surg. 2004;53(5):469472.

4. Agarwal S, Caplivski D, Bottone EJ. Disseminated tuberculosis presenting with finger swelling in a patient with tuberculosis osteomyelitis. Ann Clin Microbiol Antimicrob. 2005;3(4):18.

5. Andronikou $S$, Smith B. Spina ventosa tuberculosis dactylitis. Arch Dis Child. 2002;86(3):206.

6. Al-Qattan MM, Al-Namla A, Al-Thunayan A, Al-Omawi M. Tuberculosis of the hand. J Hand
Surg Am. 2011 ;36(8):1413-1421.

7. De Vuyst D, Vanhoenacker F, Gielen J, Bernaerts A, De Schepper AM. Imaging features of musculosketal tuberculosis. Eur Radiol. 2003;13(8):1809-1819.

8. Yoon CJ, Chunj HW, Hong SH, Kim CJ, Kang HS. MR findings of tuberculosis dactylitis: case report. Eur J Radiol. 2001 ;39(3):163-167.

9. Arakeri S, Fatima U, Ramkumar KR. Isolated Carpal and Metacarpal Bone Tuberculosis -A Case Report. Paripex Indian Journal of Research. 2014;3(3):130-132.

10. Singhal S, Arbart A, Lanjewar A, Ranjan R. Tuberculous Dactylitis- A Rare Manifestation of adult Skeletal Tuberculosis. Indian J Tuberc. 2005;52:218-219.

11. Ali N, Bhat A, Fatima A, Muzzafar K, Singh R. Tuberculous Dactylitis: A Case Series and Review of Literature. J Pioneer Med Sci. $2014 ; 4(4): 184-190$. 